\title{
openheart Estimating the extent of myocardial damage in patients with STEMI using the DETERMINE score
}

\author{
Christina Tiller, ${ }^{1}$ Magdalena Holzknecht, ${ }^{1}$ Martin Reindl, ${ }^{1}$ Ivan Lechner, ${ }^{1}$ \\ Verena Kalles, ${ }^{1}$ Felix Troger, ${ }^{2}$ Johannes Schwaiger, ${ }^{3}$ Agnes Mayr, ${ }^{2}$ Gert Klug, ${ }^{1}$ \\ Christoph Brenner, ${ }^{1}$ Axel Bauer, ${ }^{1}$ Bernhard Metzler (D) , 1 \\ Sebastian Johannes Reinstadler (iD ${ }^{1}$
}

\begin{abstract}
To cite: Tiller C, Holzknecht M, Reindl M, et al. Estimating the extent of myocardial damage in patients with STEMI using the DETERMINE score. Open Heart 2021;8:e001538. doi:10.1136/ openhrt-2020-001538
\end{abstract}

Received 11 January 2021 Revised 15 January 2021 Accepted 18 January 2021

\section{Check for updates}

(C) Author(s) (or their employer(s)) 2021. Re-use permitted under CC BY-NC. No commercial re-use. See rights and permissions. Published by BMJ.

${ }^{1}$ University Clinic of Internal Medicine III, Cardiology, Medical University of Innsbruck, Innsbruck, Tirol, Austria

${ }^{2}$ Department of Radiology I, Medical University of Innsbruck, Innsbruck, Tirol, Austria ${ }^{3}$ Department of Internal Medicine, Academic Teaching Hospital Hall in Tirol, Hall in Tirol, Austria

Correspondence to Dr Sebastian Johannes Reinstadler; sebastian. reinstadler@gmail.com

\section{ABSTRACT}

Background Recently, a simple ECG score (DETERMINE score) has been proposed for estimating myocardial scar in patients with ischaemic cardiomyopathy. We sought to evaluate the usefulness of the DETERMINE score for the assessment of myocardial infarct size (IS) as well as microvascular obstruction (MV0), in the setting of STelevation myocardial infarction (STEMI).

Methods This observational study enrolled 423 patients with STEMI (median age 56, 17\% women), revascularised by primary percutaneous coronary intervention $(\mathrm{PCl})$. For evaluation of the DETERMINE and Selvester scoring system (an established but complex ECG score for IS estimation), ECG was conducted before discharge (median 4 (IQR 2-6) days). Cardiac magnetic resonance (CMR) was conducted within a week after infarction for determination of IS and MVO.

Results Median DETERMINE score of the overall cohort was 8 points (IQR 5-11). A higher DETERMINE score was significantly associated with a larger IS ( $21 \%$ vs $11 \%$ of left ventricular myocardial mass (LVMM), $p<0.001$ ) as well as larger MVO (1.2\% vs $0.0 \%$ of LVMM, $p<0.001)$. In linear and binary multivariable logistic regression analysis, the DETERMINE score remained independently associated with IS (OR 1.09, 95\% $\mathrm{Cl} 1.02$ to $1.17, \mathrm{p}=0.014$ ) and MV0 (OR 1.12, 95\% Cl 1.04 to $1.21, p=0.003$ ), after adjustment for Selvester score and clinical indicators of IS (high-sensitivity cardiac troponin T, high-sensitivity C reactive protein, N-terminal pro-B-type natriuretic peptide, TIMI flow pre-interventional and post-interventional PCI, anterior infarct localisation).

Conclusions In patients undergoing PCI for STEMI, the DETERMINE score provides an easy and inexpensive tool for appropriate estimation of infarct severity as determined by CMR.

\section{INTRODUCTION}

The degree of myocardial tissue injury in the setting of ST-elevation myocardial infarction (STEMI) represents a main determinant of clinical outcome. ${ }^{1}$

Cardiac magnetic resonance (CMR) is the gold standard modality for non-invasive infarct severity characterisation after STEMI. ${ }^{2}$

\section{Key questions}

What is already known about this subject?

- The ECG represents the first-line diagnostic tool in patients with suspected ST-elevation myocardial infarction (STEMI). Recently, a simple ECG score (DETERMINE score), which combines $Q$ waves, fragmented QRS and inverted T waves, has been proposed for the assessment of myocardial scar after myocardial infarction. However, the usefulness of the DETERMINE score for the estimation of myocardial and microvascular injury in patients with STEMI is unknown.

What does this study add?

- The present study illustrates that the DETERMINE score is independently associated with infarct size and microvascular obstruction, determined by comprehensive cardiac MRI, in survivors of STEMI.

How might this impact on clinical practice?

- The DETERMINE score might provide as an easy and rapidly available tool for estimating the extent of myocardial as well as microvascular injury in patients with STEMI and might be used for risk stratification in the early phase after myocardial infarction.

It allows a comprehensive view on the myocardium tissue level including the detection and quantification of infarct size (IS) and microvascular obstruction (MVO) ${ }^{3}$ Both parameters have been proven to be of major prognostic relevance in patients with revascularised STEMI. ${ }^{3}$ However, in clinical practice, CMR imaging is hampered by restricted availability.

The ECG represents the first-line diagnostic tool in patients with suspected myocardial infarction and might be also useful for the estimation of myocardial infarct severity. Its universal availability and low costs in daily routine is a major advantage. In recent years, several ECG markers have been proposed as potential useful indicators of the extent 
of myocardial damage including Q-waves, ${ }^{4}$ fragmented $\mathrm{QRS}^{5}$ and T-wave inversions. ${ }^{6}$ More recently, a simple ECG score (DETERMINE score) which combines $Q$ waves, fragmented QRS and inverted $\mathrm{T}$ waves has been proposed as a promising tool for the assessment of myocardial scar in patients with a history of MI. ${ }^{7}$ However, the usefulness of the DETERMINE score for the estimation of myocardial and microvascular injury in the acute phase after MI is unknown. The purpose of the present study was, therefore, to assess the value of a simple ECG score, named the DETERMINE score, for the evaluation of myocardial as well as microvascular injury, as assessed by CMR imaging, in a well-defined cohort of patients with STEMI revascularised by primary percutaneous coronary intervention (PCI).

\section{METHODS}

\section{Study design and clinical measurements}

This observational study included 423 consecutive patients with STEMI who were prospectively enrolled in the MAgnetic Resonance IN Acute STEMI (MARINASTEMI) trial (NCT04113356). Diagnosis of STEMI was in accordance with the ESC/ACC committee criteria, ${ }^{8}$ and all patients were revascularised by primary PCI within 24 hours after symptom onset. For inclusion, first STEMI with no history of earlier MI or coronary intervention was required as well as an estimated glomerular filtration rate $>30 \mathrm{~mL} / \mathrm{min} / 1.73 \mathrm{~m}^{2}$ and Killip class $<$ III at time of CMR scan. Exclusion criteria were age below 18 years and contraindications for CMR (pacemaker, aneurysm clips, orbital foreign body, claustrophobia, known or suggested contrast agent allergy to gadolinium).

Measurements of high-sensitivity cardiac troponin $\mathrm{T}$ (hs-cTnT) were determined by using an enzyme immunoassay (hs-cTnT, E170; Roche Diagnostics, Vienna, Austria) according to the standard protocols of our working group as described previously. ${ }^{9}$ For measurements of high-sensitivity $\mathrm{C}$ reactive protein (hs-CRP), the c702 module of cobas 8000 (Roche Diagnostics) was applied. ${ }^{10} \mathrm{~N}$-Terminal pro-B-type natriuretic peptide (NT-proBNP) levels were measured by a commercially available assay (E170 instrument proBNP II assay; Roche Diagnostics).

A detailed medical history including current medications and presence of cardiovascular risk factors were assessed during hospitalisation. All patients gave written informed consent prior to study inclusion.

\section{Patient and public involvement statement}

Patients and/or the public were not involved in the design, conduct, reporting or dissemination plans of this research.

\section{Electrocardiography}

For electrocardiographic analyses, a standard 12-lead surface ECG (voltage: $10 \mathrm{~mm} / \mathrm{mV}$; speed: $25 \mathrm{~mm} / \mathrm{s}$ ) was conducted before discharge, at a median of 4 (IQR 2-6) days after the index event. DETERMINE ${ }^{7}$ and Selvester score $(37 \text { criteria/29 points })^{11}$ were evaluated manually by two experienced investigators, blinded to CMR data. DETERMINE score was defined as follows: (number of leads with $Q$ waves [x2])+(number of leads with fragmented QRS)+(number of leads with inverted $T$ waves). ${ }^{7}$ Pathological $\mathrm{Q}$ waves were defined as any $\mathrm{Q}$ wave with duration $>40 \mathrm{~ms}$ and $\mathrm{Q} / \mathrm{R}$ wave amplitude ratio $>0.25$ $\mathrm{mV}$ or absence of an $\mathrm{R}$ wave. $^{12}$ Fragmented QRS was defined by QRS duration $<120 \mathrm{~ms}$ and the RSR' pattern, specified by the presence of an additional $R$ wave $\left(R^{\prime}\right)$ or notching in the nadir of the $\mathrm{S}$ wave, or the presence of $>1 \mathrm{R}^{\prime}$ (fragmentation). ${ }^{5} \mathrm{~T}$-Wave inversion was defined as the presence of an inverted $\mathrm{T}$ wave in at least one of the infarct-related leads with the nadir deeper than 0.1 $\mathrm{mV} .{ }^{7}$ Lead aVR (augmented Vector Right) was excluded from all ECG analyses. In addition, patients with bundle branch or fascicular block and true posterior infarction were excluded $(n=18)$.

\section{Cardiac MRI}

All scans were performed on a 1.5 T MRI unit (AVANTOscanner; Siemens, Healthineers AG, Erlangen, Germany) at a median of 3 (IQR 2-5) days after infarction. A detailed imaging and post-processing protocol of our working group was published in detail previously. ${ }^{13}$ Left ventricular (LV) morphology and function were conducted on shortaxis cine images using retrospective ECG-triggered trueFISP bright-blood sequences acquired using breath hold. Standard software (ARGUS; Siemens, Healthineers AG) was used for post-processing analyses. Papillary muscles were excluded from myocardial mass and included in the $\mathrm{LV}$ volume. For LV strain analyses, a tissue tracking software was used (Circle Cardiovascular Imaging, Calgary, Canada); short-axis and long-axis images were available for 390 patients. Late gadolinium enhancement (LGE) images were acquired $15 \mathrm{~min}$ after the application of a $0.2 \mathrm{mmol} / \mathrm{kg}$ bolus of contrast agent (Gadovist; Bayer Vital, Leverkusen, Germany), using an ECG-triggered phase-sensitive inversion recovery sequence with consecutive short-axis slices. The extent of LGE was determined quantitatively on each slice using IMPAX EE workstation (Agfa HealthCare, Bonn, Germany) by defining 'hyperenhancement' at a threshold of +5 SD above the signal intensity of remote myocardium in the opposite myocardial segment of the LV. ${ }^{14}$ MVO was defined as persisting area of 'hypoenhancement' within the infarction. ${ }^{9}$ IS and MVO were expressed as percentages of LV myocardial mass (LVMM). Image analyses were performed by an established CMR core laboratory blinded to clinical and ECG data.

\section{Statistical analysis}

Continuous variables were expressed as median with corresponding IQR. Categorical variables were presented as frequencies with corresponding percentages. Differences in continuous variables were compared by the Mann-Whitney $\mathrm{U}$ test and differences in categorical variables were tested by the $\chi^{2}$ test. Kruskal-Wallis test 
was used to test differences in more than two groups. Patients were dichotomised by the median DETERMINE score (8 points). Receiver operating characteristic curve analysis was applied to evaluate the area under the curve (AUC) for the prediction of large IS (IS >19\%) and large MVO (MVO $>1.4 \%$ ). AUC values were compared according to a method characterised by DeLong et al. ${ }^{15}$ Linear and binary multivariable regression analyses were performed to assess the value of the DETERMINE score for the prediction of IS as well as MVO. For binary logistic regression analysis, IS and MVO were dichotomised by prognosis-based cut-off values (IS $>19 \%$, MVO $>1.4 \%$ ). ${ }^{16}$ For the assessment of the coefficients of variability of the DETERMINE score, the SD of differences between the calculations was divided by the mean value of these assessments. For all analyses, two-tailed $p$ values of $<0.05$ were considered to indicate statistical significance. Statistical analyses were operated with IBM SPSS Statistics V.25.0 and MedCalc V.19.0 (Ostend, Belgium).

\section{RESULTS}

\section{Baseline characteristics}

Median age of the study cohort was 56 (IQR 50-66) years and 71 patients $(17 \%)$ were female. Table 1 summarises the baseline characteristics of the entire study population and according to the median DETERMINE score.

\section{Clinical associates of the determine score}

Patients with a median DETERMINE score $\geq 8$ points had more often anterior infarct localisation (64\% vs $29 \%, \mathrm{p}<0.001)$. Regarding biomarkers, patients with a

Table 1 Patient characteristics

\begin{tabular}{|c|c|c|c|c|}
\hline & Total population $(n=423)$ & $\begin{array}{l}\text { DETERMINE score }<8 \\
(n=207)\end{array}$ & $\begin{array}{l}\text { DETERMINE score } \geq 8 \\
(\mathrm{n}=216)\end{array}$ & $P$ value \\
\hline Age, years & $56(50-66)$ & $56(49-65)$ & $57(51-66)$ & 0.159 \\
\hline Female, n (\%) & $71(17)$ & $42(20)$ & $29(13)$ & 0.059 \\
\hline Body mass index, $\mathrm{kg} / \mathrm{m}^{2}$ & $26(25-29)$ & $26(25-29)$ & $26(24-29)$ & 0.401 \\
\hline Hypertension, $\mathrm{n}(\%)$ & $212(50)$ & $98(47)$ & $114(53)$ & 0.264 \\
\hline Hyperlipidaemia, n (\%) & $239(57)$ & $121(59)$ & $118(55)$ & 0.428 \\
\hline Diabetes mellitus, $n(\%)$ & $41(10)$ & $20(11)$ & $22(10)$ & 0.727 \\
\hline Current smoker, n (\%) & $233(55)$ & $122(59)$ & $111(51)$ & 0.119 \\
\hline Family history, $\mathrm{n}(\%)$ & $144(34)$ & $76(37)$ & $68(32)$ & 0.298 \\
\hline Anterior infarct localisation, $\mathrm{n}(\%)$ & $198(47)$ & $60(29)$ & $138(64)$ & $<0.001$ \\
\hline TIMI flow 0 pre-pPCl, n (\%) & $264(62)$ & $121(59)$ & $143(66)$ & 0.100 \\
\hline TIMI flow 3 post-pPCI, n (\%) & $376(89)$ & 190 (92) & $186(86)$ & 0.063 \\
\hline Total ischaemia time, min & $186(120-307)$ & $179(108-285)$ & $195(126-340)$ & 0.124 \\
\hline Peak hs-cTnT, ng/L & $4890(2190-8574)$ & $3117(1320-5183)$ & 6957 (4388-10 900) & $<0.001$ \\
\hline Peak hs-CRP, mg/L & $23(12-46)$ & $20(9-33)$ & $28(15-57)$ & $<0.001$ \\
\hline Peak NT-proBNP, ng/L & $1165(560-2152)$ & 779 (424-1523) & $1553(758-3044)$ & $<0.001$ \\
\hline DETERMINE score & $8(5-11)$ & $5(2-6)$ & $11(9-13)$ & $<0.001$ \\
\hline Selvester score & $5(3-8)$ & $3(2-5)$ & $7(5-9)$ & $<0.001$ \\
\hline \multicolumn{5}{|l|}{ CMR parameters } \\
\hline LVEDV, mL & $148(125-170)$ & $144(121-163)$ & $154(128-178)$ & 0.003 \\
\hline LVESV, mL & $69(54-86)$ & $61(49-80)$ & $75(58-96)$ & $<0.001$ \\
\hline LVEF, \% & $53(45-59)$ & $55(49-61)$ & $50(42-56)$ & $<0.001$ \\
\hline LV global longitudinal strain, \% & $-12.0(-14.1$ to -9.7$)$ & $-13.4(-15.4$ to -11.3$)$ & $-10.8(-12.8$ to -8.8$)$ & $<0.001$ \\
\hline LV global radial strain, \% & $26.3(20.3-31.9)$ & $27.3(21.9-33.2)$ & $25.6(19.4-30.8)$ & 0.005 \\
\hline LV global circumferential strain, \% & $-14.2(-16.0$ to -11.8$)$ & $-15.0(-16.7$ to -12.9$)$ & $-13.0(-14.9$ to -10.9$)$ & $<0.001$ \\
\hline IS, \% of LVMM & $16(8-24)$ & $11(4-17)$ & $21(14-28)$ & $<0.001$ \\
\hline MV0 presence & $230(54)$ & $79(38)$ & $151(70)$ & $<0.001$ \\
\hline MVO extent, \% of LVMM & $0.4(0.0-1.9)$ & $0.0(0.0-0.9)$ & $1.2(0.0-3.6)$ & $<0.001$ \\
\hline
\end{tabular}

CMR, cardiac magnetic resonance imaging; hs-CRP, high-sensitivity C reactive protein; hs-cTnT, high-sensitivity cardiac troponin T; IS, infarct size; LVEDV, left ventricular end-diastolic volume; LVEF, left ventricular ejection fraction; LVESV, left ventricular end-systolic volume; LVMM, left ventricular myocardial mass; MVO, microvascular obstruction; NT-proBNP, N-terminal pro-B-type natriuretic peptide; pPCl, primary percutaneous coronary intervention. 


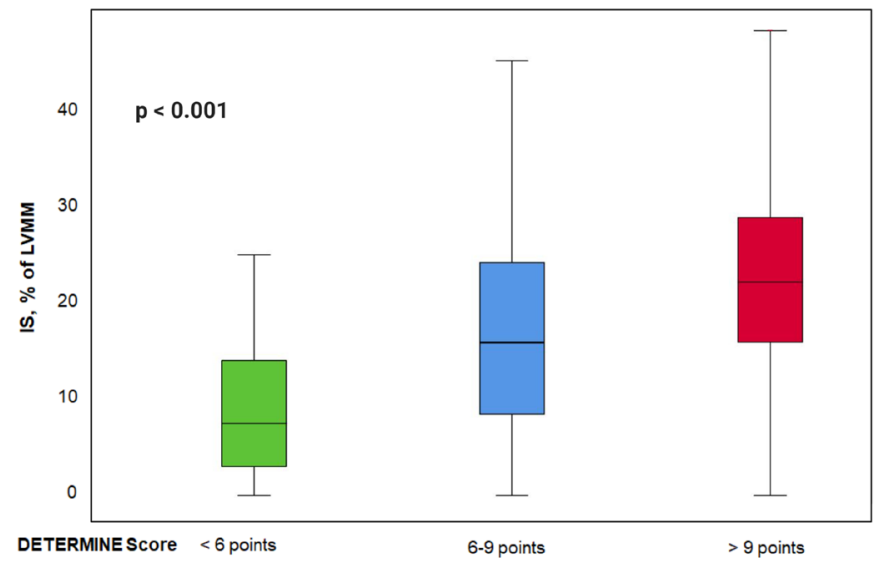

Figure 1 Relationship between the DETERMINE score ( $\mathrm{x}$ axis) and prediction of infarct size (\%,y-axis). IS, infarct size; LVMM, left ventricular myocardial mass.

DETERMINE scores $\geq 8$ points showed higher peak levels of hs-cTnT (6957 ng/L vs $3117 \mathrm{ng} / \mathrm{L}, \mathrm{p}<0.001$ ), hs-CRP (28 mg/L vs $20 \mathrm{mg} / \mathrm{L}, \mathrm{p}<0.001)$ and of NT-proBNP (1553 $\mathrm{ng} / \mathrm{L}$ vs $779 \mathrm{ng} / \mathrm{L}, \mathrm{p}<0.001$ ) during index hospitalisation. Higher DETERMINE scores were significantly related to higher Selvester score points ( 7 points vs 3 points, $\mathrm{p}<0.001)$. Regarding CMR parameters, patients with a DETERMINE score $\geq 8$ points had a significantly lower $\mathrm{LV}$ ejection fraction $(50 \%$ vs $55 \%, \mathrm{p}<0.001)$, and worse $\mathrm{LV}$ global longitudinal $(-10.8 \%$ vs $-13.4 \%, \mathrm{p}<0.001)$, radial $(25.6 \%$ vs $27.3 \%, \mathrm{p}=0.005)$ and circumferential $(-13.0 \%$ vs $-15.0 \%, \mathrm{p}<0.001)$ strain. A higher DETERMINE score was significantly associated with a larger IS (21\% vs $11 \%$ of LVMM, $\mathrm{p}<0.001)$. MVO was more frequent $(70 \%$ vs $38 \%, \mathrm{p}<0.001)$ as well as more extensive $(1.2 \%$ vs $0.0 \%$ of LVMM, $\mathrm{p}<0.001$ ) in patients with a DETERMINE score $\geq 8$ points.

Interobserver agreement was high for the DETERMINE score $(r=0.957, p<0.001)$ and corresponding coefficient of variability was $22 \%$.

\section{Utility of the scores for the assessment of myocardial injury}

Higher DETERMINE score points were related with a significant and stepwise increase in IS $(\mathrm{p}<0.001)$ : 0-5 points, 7\% (IQR 3\%-14\%); 6-9 points, 16\% (IQR $8 \%-24 \%$ ); and $>9$ points, $22 \%$ (IQR $16 \%-28 \%$ ), respectively (figure 1). AUC values of the DETERMINE score were both higher for the prediction of large IS (AUC $=0.76,95 \%$ CI 0.72 to 0.81 vs $\mathrm{AUC}=0.71,95 \%$ CI 0.66 to $0.76, \mathrm{p}=0.050$ ) as well as for the prediction of large MVO (AUC $=0.74,95 \%$ CI 0.69 to 0.79 vs $\mathrm{AUC}=0.69,95 \%$ CI 0.63 to $0.74, \mathrm{p}=0.049$ ) as compared with the Selvester score. When comparing DETERMINE score and pathological $Q$ waves, the AUC for the prediction of large IS was significantly higher for the DETERMINE score than for pathological $Q$ waves $(\mathrm{AUC}=0.76,95 \%$ CI 0.72 to 0.81 , $\mathrm{p}<0.001$ vs $\mathrm{AUC}=0.63,95 \%$ CI 0.58 to $0.68, \mathrm{p}<0.001$, AUC difference $=p<0.001)$. Also for the prediction of MVO, the DETERMINE score yielded a significantly higher AUC than pathological $\mathrm{Q}$ waves (AUC=0.74, 95\% CI 0.69 to
0.79, $\mathrm{p}<0.001$ vs $\mathrm{AUC}=0.58,95 \%$ CI 0.52 to $0.64, \mathrm{p}=0.009$, AUC difference $=\mathrm{p}<0.001$ ).

In linear regression analysis, the DETERMINE score independently predicted large IS $(\beta=0.223, p<0.001)$ and large MVO $(\beta=0.139, p=0.008)$ (table 2). Also in binary logistic regression analysis, the DETERMINE score emerged as independent predictor of large IS $(>19 \%$ of LVMM) (OR 1.09, 95\% CI 1.02 to $1.17, \mathrm{p}=0.014)$ and large MVO (OR 1.12, 95\% CI 1.04 to $1.21, \mathrm{p}=0.003$ ) after adjustment for Selvester score, hs-cTnT, hs-CRP, NT-proBNP, TIMI flow pre-interventional, and post-interventional PCI and anterior infarct localisation (table 3).

\section{DISCUSSION}

In the present study, we evaluated the utility of the DETERMINE score, a combination of $Q$ waves, fragmented QRS and inverted $\mathrm{T}$ waves, for estimating IS and MVO in a large and well-defined cohort of patients with STEMI. We could demonstrate a significant positive correlation between the DETERMINE score and IS. Furthermore, the DETERMINE score remained independently associated with IS even after adjustment for other parameters such as Selvester score, a complex ECG score that has been proposed previously for IS estimation, ${ }^{9}$ as well as established clinical parameters such as hs-cTnT, hs-CRP, NT-proBNP, TIMI flow pre-interventional, and post-interventional PCI and anterior infarct localisation. Moreover, we observed a significant and independent association of the DETERMINE score with MVO, a severe marker of reperfusion injury with major prognostic implications in patients with STEMI.

Together, these data suggest a close relation between the DETERMINE score and myocardial as well as microvascular injury as determined by CMR imaging in patients with STEMI. Therefore, this ECG score provides an easy and rapidly available tool for early infarct severity assessment post-STEMI.

\section{ECG markers and infarct size}

The magnitude of myocardial injury represents a major determinant for the prognosis after STEMI. ${ }^{2}$ CMR imaging enables a precise and comprehensive infarct characterisation ${ }^{13}$; however, it is still hampered by restricted availability and high costs. ECG offers an inexpensive and universally available tool for estimating myocardial injury after STEMI. Several promising ECG markers have been proposed for the assessment of IS. In particular, the role of pathological $Q$ waves has been studied extensively resulting in good correlation with myocardial tissue injury. ${ }^{4}{ }^{12}$ Also, the presence of fragmented QRS was demonstrated with larger areas of ischaemic injury. ${ }^{17}$ Moreover, inverted T waves after revascularisation have been illustrated as reliable ECG markers for IS estimation. ${ }^{6}$ Accordingly, evaluation of various scoring systems for IS estimation have been of great interest in the past decades. ${ }^{18} 19$ Especially the modified Selvester QRS score, a 37-criteria/29-points ECG scoring 
Table 2 Univariable and multivariable linear regression analysis for the prediction of IS and MVO

\begin{tabular}{|c|c|c|c|c|}
\hline \multicolumn{5}{|l|}{ Univariable multivariable } \\
\hline & $\beta$ & $P$ value & $\beta$ & $P$ value \\
\hline \multicolumn{5}{|l|}{ IS } \\
\hline DETERMINE score & 0.492 & $<0.001$ & 0.223 & $<0.001$ \\
\hline Selvester score & 0.386 & $<0.001$ & - & - \\
\hline Hs-cTnT, ng/L & 0.651 & $<0.001$ & 0.468 & $<0.001$ \\
\hline Peak hs-CRP, mg/L & 0.286 & $<0.001$ & - & - \\
\hline Peak NT-proBNP, ng/L & 0.330 & $<0.001$ & - & - \\
\hline TIMI flow pre-pPCI & -0.410 & $<0.001$ & -0.198 & $<0.001$ \\
\hline TIMI flow post-pPCl & -0.116 & 0.017 & - & - \\
\hline Anterior infarct localisation & 0.219 & $<0.001$ & - & - \\
\hline \multicolumn{5}{|l|}{ MVO } \\
\hline DETERMINE score & 0.352 & $<0.001$ & 0.139 & 0.008 \\
\hline Selvester score & 0.257 & $<0.001$ & - & - \\
\hline Hs-cTnT, ng/L & 0.565 & $<0.001$ & 0.506 & $<0.001$ \\
\hline Peak hs-CRP, mg/L & 0.323 & $<0.001$ & - & - \\
\hline Peak NT-proBNP, ng/L & 0.271 & $<0.001$ & - & - \\
\hline TIMI flow pre-pPCI & -0.214 & $<0.001$ & - & - \\
\hline TIMI flow post-pPCI & -0.104 & 0.033 & - & - \\
\hline Anterior infarct localisation & 0.174 & $<0.001$ & - & - \\
\hline
\end{tabular}

hs-CRP, high-sensitivity C reactive protein; hs-cTnT, high-sensitivity cardiac troponin T; IS, infarct size; MVO, microvascular obstruction; NTproBNP, N-terminal pro-B-type natriuretic peptide; pPCl, primary percutaneous coronary intervention.

Table 3 Binary logistic regression analysis for the prediction of IS and MVO

\begin{tabular}{|c|c|c|c|c|}
\hline & \multicolumn{2}{|c|}{ Univariable analysis } & \multicolumn{2}{|c|}{ Multivariable analysis } \\
\hline & OR (95\% CI) & $P$ value & OR (95\% Cl) & $P$ value \\
\hline \multicolumn{5}{|l|}{ IS $>19 \%$} \\
\hline DETERMINE score & 1.30 (1.23 to 1.39$)$ & $<0.001$ & 1.09 (1.02 to 1.17$)$ & 0.014 \\
\hline Selvester score & 1.26 (1.18 to 1.35$)$ & $<0.001$ & - & - \\
\hline Hs-cTnT, ng/L & 1.00 (1.00 to 1.00$)$ & $<0.001$ & 1.00 (1.00 to 1.00$)$ & $<0.001$ \\
\hline Peak hs-CRP, mg/L & 1.14 (1.08 to 1.20$)$ & $<0.001$ & - & - \\
\hline Peak NT-proBNP, ng/L & 1.00 (1.00 to 1.00$)$ & $<0.001$ & - & - \\
\hline TIMI flow 0 pre-pPCl & 0.21 (0.13 to 0.34 ) & $<0.001$ & 0.40 (0.22 to 0.74$)$ & 0.003 \\
\hline TIMI flow 3 post-pPCI & 0.35 (0.19 to 0.66$)$ & 0.001 & - & - \\
\hline Anterior infarct localisation & 2.04 (1.37 to 3.04 ) & $<0.001$ & - & - \\
\hline \multicolumn{5}{|l|}{ MVO $>1.4 \%$} \\
\hline DETERMINE score & 1.22 (1.16 to 1.29$)$ & $<0.001$ & 1.12 (1.04 to 1.21$)$ & 0.003 \\
\hline Selvester score & 1.21 (1.14 to 1.29$)$ & $<0.001$ & - & - \\
\hline Hs-cTnT, ng/L & 1.00 (1.00 to 1.00$)$ & $<0.001$ & 1.00 (1.00 to 1.00$)$ & $<0.001$ \\
\hline Peak hs-CRP, mg/L & 1.17 (1.11 to 1.24$)$ & $<0.001$ & 1.13 (1.07 to 1.20$)$ & $<0.001$ \\
\hline Peak NT-proBNP, ng/L & $1.00(1.00$ to 1.00$)$ & $<0.001$ & - & - \\
\hline TIMI flow 0 pre-pPCl & 0.29 (0.18 to 0.48$)$ & $<0.001$ & 0.52 (0.28 to 0.95$)$ & 0.033 \\
\hline TIMI flow 3 post-pPCI & 0.40 (0.22 to 0.75$)$ & 0.004 & - & - \\
\hline Anterior infarct localisation & 1.89 (1.24 to 2.88 ) & 0.003 & - & - \\
\hline
\end{tabular}

hs-CRP, high-sensitivity C reactive protein; hs-cTnT, high-sensitivity cardiac troponin T; IS, infarct size; MVO, microvascular obstruction; NTproBNP, N-terminal pro-B-type natriuretic peptide; pPCl, primary percutaneous coronary intervention. 
system, has been suggested for IS assessment. ${ }^{9}{ }^{20}$ However, it has recently been shown that the Selvester QRS score only has moderate association to CMR-determined IS. ${ }^{9}$ Moreover, due to the numerous criteria that have to be fulfilled for the Selvester score, this score is hardly applicable in clinical practice. Therefore, Lee $e t$ al introduced a simple ECG score based on the presence of abnormal ECG markers (combination of $Q$ waves, fragmented QRS and inverted $\mathrm{T}$ waves) to estimate myocardial scar. ${ }^{7}$ They demonstrated in a cohort of 551 patients with ischaemic cardiomyopathy that the DETERMINE score estimated IS nearly as good as LVEF measured by CMR. ${ }^{7}$ However, due to the very large time gap between infarction and the ECG (median 5 years), the DETERMINE score might differ significantly if analysed in the acute setting after infarction because of the dynamic changes of the individual ECG markers especially in the early phase postSTEMI. Furthermore, CMR imaging was performed within 40 days after infarction, which is also a very large time gap between infarction and imaging since the most common time point for infarct severity assessment by CMR imaging is performed between days 3 and $5 .{ }^{21}$ Moreover, patients were excluded if the infarct mass was $<10 \%$ in CMR imaging. The present analysis describes for the first time a close correlation of the DETERMINE score with IS and MVO determined by CMR in a large cohort of patients with STEMI. Above all, the DETERMINE score was independently associated with IS even after adjustment for Selvester score, hs-cTnT, hs-CRP, NT-proBNP, TIMI flow pre-interventional, and post-interventional PCI and anterior infarct localisation. To foreground, the AUC of the DETERMINE score was higher for the prediction of large IS as compared with the Selvester score. Thus, the DETERMINE score is a reliable tool for the exact estimation of myocardial damage after STEMI.

\section{ECG markers and MVO}

MVO, a severe marker of reperfusion injury, is common after STEMI treated with primary PCI with a prevalence of up to $50 \% .^{22}$ Presence of MVO is associated with worse LV function, larger IS and subsequently with higher risk of recurrent cardiovascular events. ${ }^{23}$ Identification of MVO is, therefore, of high relevance to allow an optimal risk stratification in the early stage after STEMI. CMR imaging currently offers the best tool in quantifying the presence and extent of MVO, ${ }^{24}$ however limited due to high costs and availability. The relation of ECG parameters with MVO assessed by CMR are scarce in the current literature either due to small study cohorts or using other reference methods for MVO quantification rather than CMR. Recently, we showed that patients with pathological $\mathrm{Q}$ waves on admission show more extensive MVO. Rommel et al demonstrated that QRS distortion on the admission ECG is significantly associated with MVO. ${ }^{25}$ In addition, patients displaying T-wave inversions significantly showed higher rates of MVO. ${ }^{6}$ In line with those prior findings, the DETERMINE score, a combination of the latter mentioned ECG markers, revealed a significant association with the presence as well as the extent of MVO. Accordingly, our results underline the value of the DETERMINE score for IS estimation and also for estimating microvascular injury in STEMI survivors.

\section{Clinical implications}

The DETERMINE score provides an almost simple and immediately available ECG score, combining established patterns of $\mathrm{Q}$ waves, fragmented QRS and inverted $\mathrm{T}$ waves, for IS estimation and assessment of MVO. In multivariable analysis, the DETERMINE score revealed a significant and independent association with both large IS and presence as well as extent of MVO even after adjustment for other established clinical parameters. However, further research is needed to evaluate the clinical applicability of the DETERMINE score in patients with STEMI.

\section{Limitations}

Our study has to declare some limitations. First, due to the inclusion of stable patients with STEMI with Killip class $<3$, these findings may not be applicable to unstable patients. Of note, the vast majority of patients with STEMI represent with Killip class $<3 .{ }^{26}$ Second, CMR imaging was performed at a median of 3 days which might overestimate acute IS; however, in a recent scientific expert panel, CMR imaging is recommended $5 \pm 2$ days after reperfusion. ${ }^{2}$ In addition, salvaged myocardium was not evaluated in the present analysis, primarily due to lack of T2-weighted validated data. ${ }^{27}$ Third, the DETERMINE score should be validated in an independent STEMI cohort to prove the results from the present data for clinical applicability. Fourth, the DETERMINE score was analysed at discharge, thus, the predictive value may differ if measured at other time points. Although persistent ST elevation represents a marker of IS and MVO, it was not included in the DETERMINE score. Further studies are necessary to evaluate the comparative value of persistent ST elevation and the DETERMINE score. Finally, pathological $Q$ waves were used as a dichotomous variable as required for the DETERMINE score, although recent data suggest that continuous variable of $Q$ waves might show better association with IS as well as MVO. ${ }^{28}$ Furthermore, we cannot fully exclude other confounders resulting in pathological $\mathrm{Q}$ waves; however, according to our exclusion criteria, patients with known history of cardiac diseases were excluded.

\section{CONCLUSION}

A simple ECG score, representing a combination of $Q$ waves, fragmented QRS and T-wave inversions, ascertained from the discharge ECG, is independently associated with the extent of myocardial injury after STEMI. Thus, the DETERMINE score may represent as a reliable, and rapidly available tool for estimating the extent of myocardial as well as microvascular injury. 
Acknowledgements This study was supported by grants from the 'Austrian Society of Cardiology', the 'Tiroler Wissenschaftsfonds' and by the 'Austrian Science Fund' (FWF): KLI 772-B.

Contributors CT: planning, conducting; data collection and reporting; statistical analysis, article writing, submission. $\mathrm{MH}$ : patient enrolment, data collection, article check. MR: article and statistical check. IL: patient enrolment, article check. VK: patient enrolment. FT: patient enrolment. JS: data collection, reporting, article check. AM: CMR analysis. GK: statistical check, CMR analysis. CB: data and article check. AB: data check and proof reading. BM: data, statistical and article check. SJR: planning, reporting, article writing.

Disclaimer The funders had no role in study design, in collection or interpretation of data, or in writing the manuscript.

Competing interests None declared.

Patient consent for publication Not required.

Ethics approval The study was approved by the local ethics committee of the Medical University of Innsbruck (reference number AN3775 281/4.15) in concordance with the Declaration of Helsinki.

Provenance and peer review Not commissioned; externally peer reviewed.

Data availability statement Data are available on reasonable request.

Open access This is an open access article distributed in accordance with the Creative Commons Attribution Non Commercial (CC BY-NC 4.0) license, which permits others to distribute, remix, adapt, build upon this work non-commercially, and license their derivative works on different terms, provided the original work is properly cited, appropriate credit is given, any changes made indicated, and the use is non-commercial. See: http://creativecommons.org/licenses/by-nc/4.0/.

ORCID iDs

Bernhard Metzler http://orcid.org/0000-0002-5240-2453

Sebastian Johannes Reinstadler http://orcid.org/0000-0002-7700-1357

\section{REFERENCES}

1 Reinstadler SJ, Thiele H, Eitel I. Risk stratification by cardiac magnetic resonance imaging after ST-elevation myocardial infarction. Curr Opin Cardiol 2015;30:681-9.

2 Ibanez B, Aletras AH, Arai AE, et al. Cardiac MRI endpoints in myocardial infarction experimental and clinical trials: JACC Scientific Expert Panel. J Am Coll Cardiol 2019;74:238-56.

3 Reinstadler SJ, Stiermaier T, Fuernau G, et al. The challenges and impact of microvascular injury in ST-elevation myocardial infarction. Expert Rev Cardiovasc Ther 2016;14:431-43.

4 Tiller C, Reindl M, Holzknecht M, et al. Relationship between admission $\mathrm{Q}$ waves and microvascular injury in patients with STelevation myocardial infarction treated with primary percutaneous coronary intervention. Int J Cardiol 2019;297:1-7.

5 Das MK, Khan B, Jacob S, et al. Significance of a fragmented QRS complex versus a $Q$ wave in patients with coronary artery disease. Circulation 2006;113:2495-501.

6 Reindl M, Reinstadler SJ, Feistritzer H-J, et al. Persistent T-wave inversion predicts myocardial damage after ST-elevation myocardial infarction. Int J Cardiol 2017;241:76-82.

7 Lee DC, Albert CM, Narula D, et al. Estimating myocardial infarction size with a simple electrocardiographic marker score. J Am Heart Assoc 2020;9:e014205.

8 Thygesen K, Alpert JS, Jaffe AS, et al. Third universal definition of myocardial infarction. Eur Heart J 2012;33:2551-67.

9 Tiller C, Reindl M, Reinstadler SJ, et al. Complete versus simplified Selvester QRS score for infarct severity assessment in ST-elevation myocardial infarction. BMC Cardiovasc Disord 2019;19:285.
10 Tiller C, Reindl M, Holzknecht M, et al. Biomarker assessment for early infarct size estimation in ST-elevation myocardial infarction. Eur J Intern Med 2019;64:57-62.

11 Bounous EP, Califf RM, Harrell FE, et al. Prognostic value of the simplified Selvester QRS score in patients with coronary artery disease. J Am Coll Cardiol 1988;11:35-41.

12 Delewi R, ljff $\mathrm{G}$, van de Hoef TP, et al. Pathological Q waves in myocardial infarction in patients treated by primary $\mathrm{PCI}$. JACC Cardiovasc Imaging 2013;6:324-31.

13 Reinstadler SJ, Klug G, Feistritzer H-J, et al. Prognostic value of left ventricular global function index in patients after ST-segment elevation myocardial infarction. Eur Heart $J$ Cardiovasc Imaging 2016;17:169-76.

14 Bondarenko O, Beek AM, Hofman MBM, et al. Standardizing the definition of hyperenhancement in the quantitative assessment of infarct size and myocardial viability using delayed contrast-enhanced CMR. J Cardiovasc Magn Reson 2005;7:481-5.

15 DeLong ER, DeLong DM, Clarke-Pearson DL. Comparing the areas under two or more correlated receiver operating characteristic curves: a nonparametric approach. Biometrics 1988;44:837-45.

16 Eitel I, de Waha S, Wöhrle J, et al. Comprehensive prognosis assessment by CMR imaging after ST-segment elevation myocardial infarction. J Am Coll Cardiol 2014;64:1217-26.

17 Ma X, Duan W, Poudel P, et al. Fragmented QRS complexes have predictive value of imperfect ST-segment resolution in patients with STEMI after primary percutaneous coronary intervention. Am J Emerg Med 2016;34:398-402.

18 Seino Y, Staniloff HM, Shell WE, et al. Evaluation of a QRS scoring system in acute myocardial infarction: relation to infarct size, early stage left ventricular ejection fraction, and exercise performance. $\mathrm{Am}$ J Cardiol 1983;52:37-42.

19 Pahlm US, Chaitman BR, Rautaharju PM, et al. Comparison of the various electrocardiographic scoring codes for estimating anatomically documented sizes of single and multiple infarcts of the left ventricle. Am J Cardiol 1998;81:809-15.

20 Wagner GS, Freye CJ, Palmeri ST, et al. Evaluation of a QRS scoring system for estimating myocardial infarct size. I. Specificity and observer agreement. Circulation 1982;65:342-7.

21 Dall'Armellina E, Karia N, Lindsay AC, et al. Dynamic changes of edema and late gadolinium enhancement after acute myocardial infarction and their relationship to functional recovery and salvage index. Circ Cardiovasc Imaging 2011;4:228-36.

22 van Kranenburg M, Magro M, Thiele $\mathrm{H}$, et al. Prognostic value of microvascular obstruction and infarct size, as measured by CMR in STEMI patients. JACC Cardiovasc Imaging 2014;7:930-9.

23 Hamirani YS, Wong A, Kramer CM, et al. Effect of microvascular obstruction and intramyocardial hemorrhage by CMR on LV remodeling and outcomes after myocardial infarction: a systematic review and meta-analysis. JACC Cardiovasc Imaging 2014;7:940-52.

24 Klug G, Metzler B. Assessing myocardial recovery following STsegment elevation myocardial infarction: short- and long-term perspectives using cardiovascular magnetic resonance. Expert Rev Cardiovasc Ther 2013;11:203-19.

25 Rommel K-P, Badarnih H, Desch S, et al. QRS complex distortion (grade 3 ischaemia) as a predictor of myocardial damage assessed by cardiac magnetic resonance imaging and clinical prognosis in patients with ST-elevation myocardial infarction. Eur Heart $J$ Cardiovasc Imaging 2016;17:194-202.

26 El-Menyar A, Zubaid M, AlMahmeed W, et al. Killip classification in patients with acute coronary syndrome: insight from a multicenter registry. Am J Emerg Med 2012;30:97-103.

27 Friedrich MG, Kim HW, Kim RJ. T2-weighted imaging to assess post-infarct myocardium at risk. JACC Cardiovasc Imaging 2011;4:1014-21.

28 Topal DG, Lønborg J, Ahtarovski KA, et al. Early Q-wave morphology in prediction of reperfusion success in patients with ST-segment elevation myocardial infarction treated with primary percutaneous coronary intervention - a cardiac magnetic resonance imaging study. J Electrocardiol 2020;58:135-42. 